Sola Reche, J.M., Marín Marín, J.A., Alonso García, S. \& Gómez García, G. (2020). Análisis de percepciones del estudiantado del Máster de Secundaria respecto a las competencias profesionales del docente. Revista Electrónica Interuniversitaria de Formación del Profesorado, 23(2), 81-93.

DOI: https://doi.org/10.6018/reifop.418601

\title{
Análisis de percepciones del estudiantado del Máster de Secundaria respecto a las competencias profesionales del docente
}

\author{
José María Sola Reche1, José Antonio Marín Marín², Santiago Alonso García², Gerardo \\ Gómez García ${ }^{2}$ \\ ${ }^{(1)}$ Universidad de Alicante; $\left(^{2}\right)$ Universidad de Granada \\ Grupo de investigación AREA (HUM-672)
}

\section{Resumen}

En la actualidad, la formación en competencias profesionales docentes constituye un pilar básico y fundamental a la hora de llevar a cabo la formación inicial del profesorado. Es fundamental que desde la Educación Superior se promueva una praxis educativa que permita que los aspirantes a futuros profesores conozcan las múltiples demandas que el docente debe desempeñar con eficacia en el contexto escolar. En base a esta idea, el presente trabajo tiene el objetivo de analizar las percepciones de 410 estudiantes del máster de profesorado de Educación Secundaria acerca de las competencias profesionales que debe reunir un docente. Para ello, se abogó por una metodología transversal descriptiva y correlacional, utilizando como instrumento de medida el cuestionario. Los resultados mostraron que los estudiantes otorgan gran valor a aquellas cuestiones vinculadas a la competencia aprender a aprender, la importancia de la adecuada convivencia y relaciones sociales, así como el fomento de un espíritu crítico y reflexivo. Asimismo, se establecieron relaciones correlaciónales entre aquellas variables vinculadas a la enseñanza emocional y a los ámbitos tutoriales y de orientación. Por lo tanto, es preciso continuar en una línea de trabajo que siga en la mejora de la formación inicial de futuros docentes ante los numerosos retos que le deparan en el contexto educativo actual.

\section{Palabras clave}

Competencias profesionales; profesorado; Educación Secundaria; Formación del profesorado.

\section{Contacto:}

Gerardo Gómez García, gomezgarcia@ugr.es, Campus Universitario de Cartuja s/n 18071, Granada. 


\title{
Analysis of the expectations of the students of the Master of Secondary Education with regard to the professional competences of the teacher
}

\begin{abstract}
At current, training in professional teaching skills is a basic and fundamental pillar in carrying out initial teacher training. It is essential that Higher Education promotes an educational practice that allows aspiring teachers to be aware of the multiple demands that the teacher must carry out effectively in the school context. Based on this idea, the present work aims to analyse the perceptions of 410 students of the Master's Degree in Secondary Education about the professional skills that a teacher should have. To this end, a descriptive and correlational transversal methodology was advocated, using the questionnaire as a measurement instrument. The results showed that students attach great value to those issues linked to the learning to learn competence, the importance of proper coexistence and social relations, as well as the promotion of a critical and reflective spirit. Likewise, correlation was established between those variables linked to emotional teaching and to the tutorial and guidance areas. Therefore, it is necessary to continue in a line of work that continues to improve the initial training of future teachers in the face of the many challenges that they face in the current educational context.
\end{abstract}

\section{Key words}

Professional skills; teachers; secondary education; teacher training.

\section{Introducción}

La figura del docente ha sido objetivo de la investigación educativa a lo largo de los años. Son múltiples las funciones y los ámbitos a abarcar dentro del desempeño que lleva a cabo, y conocerlas se presenta como una necesidad a abarcar dentro de la formación del profesorado. Así, desde hace décadas existe un consenso común en la necesidad de definir aquellas competencias docentes, así como en la necesidad de promover su conocimiento y mejora en la formación inicial de futuros profesores (Pesquero et al, 2008).

Con el paso de los años, la sociedad demanda con más fuerza que la formación de profesionales que no solo sean capaces de resolver con eficiencia problemas de la práctica profesional, sino también lograr un desempeño óptimo con sus estudiantes. Es decir, la sociedad de la actualidad demanda docentes que sean competentes. El término competencia constituye también un concepto integrado porque unifica en un solo término, los elementos ejecutivos e inductivos de la autorregulación de la personalidad dentro del proceso docente-educativo (Moreno, Casanova, Socarrás, Álvarez y Tabares, 2019; Acebedo, Aznar e Hinojo, 2017).

En la actualidad, son múltiples las funciones que debe de realizar el docente en su día a día, como son: adaptar el marco curricular a la realidad del aula; conocer enfoques metodológicos atractivos que sean capaces de motivar al estudiantado y transmitir correctamente la enseñanza; promover un clima adecuado en el aula, favorecedor de la participación y la óptima convivencia; promover estrategias de orientación personal, académica y profesional o; ejercer una acción tutorial responsable entre los más destacados (Martín, Pastor y Oliva, 2019). 
En general, tal y como define Ortiz (2002) las competencias profesionales pueden ser definidas como aquellas cualidades de la personalidad que permiten la autorregulación de la conducta del sujeto a partir de la integración de los conocimientos científicos, las habilidades y las capacidades vinculadas con el ejercicio de su profesión, así como los motivos, sentimientos, necesidades y valores asociados a ellas, que permiten, faciliten y promuevan un desempeño profesional eficaz y eficiente dentro de un contexto social determinado. Expresan un enfoque holístico de la personalidad en la unidad de lo cognitivo, afectivo y conductual.

Es por ello, que desde las enseñanzas superiores, la formación debe caracterizarse por promover e incentivar la capacidad para actuar, descubrir, razonar, crear, tomar decisiones, analizar la información con sentido crítico y conocer, desde la mayor contextualización posible el panorama futuro en el que los estudiantes se van a encontrar. Asimismo, debe tratarse de una formación que abarque su foco en la contextualización de los aprendizajes (Paiva, Romero y Gómez, 2018).

El Máster oficial de Profesorado de Educación Secundaria Obligatoria y Bachillerato, Formación profesional y Enseñanza de Idiomas es desde el curso académico 2009/2010 el equivalente a lo que anteriormente era conocido como CAP (Curso de Aptitud Pedagógica). Dicho máster se implantó tras descartar diversas opciones como el Curso de Cualificación Pedagógica o el Título de Especialización Didáctica, asociados ambos en un primer momento a la LOGSE (Ley Orgánica 1/1990, de 3 de octubre, de Ordenación General del Sistema Educativo), y más tarde a la LOCE (Ley Orgánica 10/2002, de 23 de diciembre, de Calidad de la Educación). Probablemente, podríamos afirmar que esta fluctuación desde el CAP hasta la implantación del máster que tenemos en la actualidad, se ha debido al descrédito que ha sufrido el primero con el paso de los años. Las causas de esto último son numerosas, pero como mantiene Rego y Moledo (2015), encontramos que la didáctica era ignorada en el mejor de los casos, mientras que no se encontraba nada que se asemejara a la pedagogía en dicho curso, por lo que su largo desarrollo en el tiempo ha provocado un desprestigio compartido por toda la comunidad educativa hasta que se ha hallado la alternativa.

La finalidad de este máster es proporcionar una formación inicial de calidad al futuro profesorado de secundaria, preparándolo para los desafíos de una sociedad cambiante donde el sistema educativo juega un papel fundamental adaptándose y dando respuesta a las nuevas tendencias que van surgiendo. Siguiendo a Terigi (2009), observamos que la relación existente entre la escuela secundaria presente y la deseada es un asunto de primer orden, del mismo modo, lo es cómo influye la formación de los docentes a la preservación del statu quo en este nivel educativo. Es evidente que no se puede plantear un cambio notable y eficaz en los sistemas formadores de los docentes, en este caso el máster que habilita para ser docente en Educación Secundaria, si no se da previamente una renovación en el currículum y en cómo el profesorado implementa y lleva a cabo el mismo.

La elección de hacer una carrera docente puede deberse a diferentes motivos, entre los que se enmarcan las expectativas que tiene este alumnado que decide realizar el máster habilitador para impartir clase en Educación Secundaria. Dichas expectativas pueden ser fundamentalmente de dos tipos (Kass y Miller, 2018):

- Por un lado, encontraríamos unas motivaciones extrínsecas como por ejemplo el conocimiento del salario u otro tipo de beneficios que se presupongan que un docente tiene en esa profesión, un estatus y un prestigio en la sociedad por parte de estas personas, etc. 
- Por otro lado, estarían las motivaciones intrínsecas, tal y como Friedman (2016) las establece pueden ser de diversa índole: las expectativas altruistas y las narcisistas del futuro rol que se pretende tener como docente.

Es de vital importancia no caer en la confusión que suscitan los términos aspiraciones y expectativas. En primera instancia, el término aspiraciones hace referencia a todo lo referente a esperanzas y sueños y no tienen por qué tener una relación directa con la posición social y económica del estudiante, así como su experiencia y vivencia pasada y actual en el ámbito educativo; mientras que, las expectativas sí están conectadas en un mayor grado con la situación socioeconómica del estudiante y, por otro lado, son un magnífico elemento para pronosticar el rendimiento académico (Khattab, 2015).

Las expectativas que los estudiantes tienen acerca de su futuro y de la formación que determinado programa de estudios va a proporcionarles es una de las fuentes de información más valiosas (Sander et al., 2000) y provechosas existentes, en lo que concierne a saber qué quieren los alumnos, qué pretenden llegar a alcanzar y cómo piensan o se sienten con respecto a los estudios que están realizando. Siguiendo a Voss et al. (2007), es imposible no reconocer las ventajas que nos aporta ser conocedores de las expectativas que tiene un determinado grupo de alumnos, no solo sus expectativas generales ante su carrera profesional y de vida, sino sus expectativas sobre temas académicos, y es aquí donde, bajo nuestro punto de vista, está el elemento más importante si nos centramos en la mejora del máster a través del feedback que nos proporciona el alumnado. Encontramos entonces dos elementos que se interconectan y se retroalimentan, puesto que son las expectativas del alumnado a través de donde podemos conocer su opinión fundamentada y su valoración con respecto a la calidad docente, los métodos empleados para impartir clases y el contenido del curso, ya sea este didáctico o no, entre otras muchas cosas, pero al mismo tiempo estas expectativas, y por ende sus valores, están influenciados, generando en ocasiones cambios en los mismos, por el servicio educativo y la calidad de este que les es ofrecido (Hill, 1995; Telford y Masson, 2005).

Sin embargo, son las percepciones con respecto a las competencias profesionales las que nos llevan a conocer qué elementos son más importantes para el futuro profesorado a la hora de llevar a cabo el proceso de enseñanza-aprendizaje (E-A). A este respecto, cabe subrayar que las competencias profesionales han sido objeto de discusión y estudio durante muchos años, por eso ser consciente de en qué elementos ponen la lupa los alumnos del máster y cuáles son vagamente tomados en cuenta nos va a permitir determinar en qué aspectos habría que incidir para que en una futura práctica docente sean elementos conductores de las clases de Educación Secundaria. Teniendo en cuenta en el mundo que nos encontramos y los retos que nos presenta el sistema educativo, así como la sociedad en general, nuestro propósito es conocer las expectativas en cuanto a las competencias profesionales docentes que el alumnado del máster considera que debe tener en su capacitación pedagógica. Atendiendo a lo establecido por Muñoz (2003), las competencias profesionales que debe desarrollar un docente son:

- Planificación de cursos, conociendo tanto al grupo a nivel general como a cada alumno a nivel individual.

- Diseño de estrategias de E-A adaptadas a las características de los participantes y el contexto.

- Búsqueda, preparación e implementación de recursos y materiales.

- Comunicación con los alumnos.

- Motivación del alumnado. 
- Propiciamiento de la participación del alumnado en clase.

- Asesoramiento y orientación a través de todo el proceso de E-A.

- Implicación y evaluación en todos los niveles del proceso de E-A.

- Fomento de una actitud crítica y reflexiva.

Este estudio es de interés principal e inmediato no solo para aquellas personas que están cursando o han cursado el máster y se dedican a la docencia en Educación Secundaria, sino para aquellas encargadas de diseñarlo y de enfocarlo tal y como se hace. En un contexto más amplio, todas aquellas personas vinculadas directa o indirectamente con la enseñanza a estos niveles o en niveles universitarios podrían tomar este documento como referencia para posibles decisiones o reflexiones al respecto.

Debemos aceptar que la competencia profesional es un saber complejo, puesto que implica un conjunto amplio de saberes $y$, por tanto, evaluar la competencia profesional no se reduce a un único curso o una única asignatura en la cual se adquiere dicha competencia. Estamos, como ya hemos dicho, ante un conjunto de saberes, los cuales deben ser integrados y adquiridos a lo largo del tiempo. Pon ende, la evaluación de la competencia profesional conlleva un enfoque interdisciplinar (Fernández y Bueno, 2016).

En este sentido, Lauermann y Konig (2016), proponen cómo un elemento básico a nivel cognitivo que debe estar dentro de la competencia profesional docente es un conocimiento pedagógico y didáctico general; en contraposición a los conocimientos específicos de las asignaturas, aquí nos estamos refiriendo a un conocimiento orientado a aspectos relativos a los procesos instruccionales, a la correcta gestión de la clase, la planificación teniendo en cuenta las necesidades de todos los alumnos, etc. Podemos ver como la interdisciplinariedad es el elemento conductor de esta competencia y su evaluación.

Sin embargo, algunas investigaciones llevadas a cabo sobre la temática de las competencias, en este caso las docentes (Konig, 2013), nos muestran cómo por un lado se percibe que un contenido general en pedagogía y didáctica en los programas educativos y formativos resulta poco útil mientras no se adapte a las necesidades de los futuros docentes, por lo que se precisa de una reestructuración. Aunque, sí cabe señalar, que en esta misma investigación se demuestra cómo sí es percibida como positiva y necesaria una formación teórica antes de producirse la formación práctica, para llegar al desarrollo completo de la competencia profesional.

\section{Metodología}

Tras el análisis de la literatura especializada, se abogó por llevar a cabo un diseño transversal, a través de un diseño metodológico cuantitativo descriptivo e inferencial (Hernández, Fernández y Baptista, 2016; López-Roldán y Fachelli, 2015), seleccionando el cuestionario como técnica de recogida de datos. Con ello, se pretende conocer la opinión de los estudiantes de las distintas especialidades que oferta el Máster oficial de Profesorado de Educación Secundaria Obligatoria y Bachillerato, Formación profesional y Enseñanza de Idiomas de la Universidad de Granada acerca de distintas competencias profesionales que debe tener un docente. Asimismo, también se indagará en conocer la naturaleza de la distribución de datos, para analizar la comparación entre medias a través de pruebas científicas reconocidas.

\section{Participantes}

La población está constituida por los estudiantes de posgrado que cursan el máster de Educación Secundaria en la Universidad de Granada en el curso de 2019-2020. La muestra 
objeto de estudio está conformada por un total de 410 alumnos de distintas menciones del Máster de Secundaria, de la cual un $35,1 \%$ son hombres y un $64,9 \%$ son mujeres. Respecto al tramo de edad, este resulta variado, diferenciando distintos intervalos; un 63,3\% tiene entre 21 a 25 años, un $26,5 \%$ pertenece al tramo entre 26 y 30 años, un 4,8 entre 31 a 35, un 3,5\% que comprende entre 36 y 40 años y un $1,6 \%$ restante que pertenece al intervalo de más de 40 años. El procedimiento a través del cual se llevó a cabo la confección de la muestra fue un muestreo aleatorio por conglomerados, en el de las 15 especialidades que consta el máster, se abogó por la elección al azar de siete de grupos, hasta llevar a un tamaño muestral significativo, con un intervalo de confianza del $95 \%$ y un margen de error del $5 \%$ (Otzen y Manterola, 2017).

\section{Instrumento}

Para la recogida de datos, se procedió a la elaboración de un cuestionario ad hoc acerca de las percepciones que tienen el alumnado del Máster de Educación Secundaria acerca de competencias profesionales del docente. Para su configuración, se tomó como referencia trabajos precedentes que utilizaron instrumentos de similar naturaleza (Ramírez, de Pozo, \& Porlán, 2013; Serrano, \& Pontes, 2015). Atendiendo a su tipología, se trata de una escala tipo Likert de cuatro niveles (1: Mucho 2: Bastante, 3: Regular y 4: Poco) con un total de 13 variables dependientes. Asimismo, se añadieron cuestiones sobre datos sociodemográficos: género, edad y grupo de clase.

La opción de respuesta oscila entre 1 y 4 , siendo Los ítems que conforman el instrumento son los siguientes:

- Ítem 1: Conocer los contenidos curriculares de las materias relativas a la especialización docente correspondiente, así como el cuerpo de conocimientos didácticos en torno a los procesos de enseñanza y aprendizaje respectivos.

- Ítem 2: Planificar, desarrollar y evaluar el proceso de enseñanza y aprendizaje potenciando procesos educativos que faciliten la adquisición de las competencias propias de las respectivas enseñanzas, atendiendo al nivel y formación previa de los estudiantes.

- Ítem 3: Buscar, obtener, procesar y comunicar información (oral, impresa, audiovisual, digital o multimedia), transformarla en conocimiento y aplicarla en los procesos.

- Ítem 4: Concretar el currículo que se vaya a implantar en un centro docente participando en la planificación colectiva del mismo; desarrollar y aplicar metodologías.

- Ítem 5: Diseñar y desarrollar espacios de aprendizaje con especial atención a la equidad, la educación emocional y en valores, la igualdad de derechos y oportunidades.

- Ítem 6: Adquirir estrategias para estimular el esfuerzo del estudiante y promover su capacidad para aprender por sí mismo y con otros, y desarrollar habilidades de pensamiento y de decisión que faciliten la autonomía, la confianza e iniciativa personales.

- Ítem 7: Conocer los procesos de interacción y comunicación en el aula, dominar destrezas y habilidades sociales necesarias para fomentar el aprendizaje y la convivencia.

- Ítem 8: Desarrollar las funciones de tutoría y de orientación de los estudiantes de manera colaborativa y coordinada; participar en la evaluación, investigación y la innovación de los procesos de enseñanza y aprendizaje.

- Ítem 9: Conocer la normativa y organización institucional del sistema educativo y modelos de mejora de la calidad con aplicación a los centros de enseñanza.

- Ítem 10: Conocer y analizar las características históricas de la profesión docente, su situación actual, perspectivas e interrelación con la realidad social de cada época. 
- Ítem 11: Informar y asesorar a las familias acerca del proceso de enseñanza y aprendizaje y sobre la orientación personal, académica y profesional de sus hijos.

- Ítem 12: Fomentar el espíritu crítico, reflexivo y emprendedor.

- Ítem 13: Fomentar y garantizar el respeto a los Derechos Humanos y a los principios de accesibilidad universal, igualdad, no discriminación y los valores democráticos y de la cultura de la paz.

El cuestionario fue sometido a un proceso de validación de contenido por juicio de expertos, compuesto por diferentes doctores de diferentes universidades. Además, llevó a cabo la validación factorial a través del cálculo de coeficiente Kaiser-Meyer-Olkin para la adecuación del muestreo $(\mathrm{KMO}=.809)$ y la prueba de esfericidad de Barlett $\left(\chi^{2}=1568.83\right.$; $\mathrm{df}$ $=78 ; p$-value $=.000$ )

Finalmente, respecto a la validez y consistencia interna del instrumento, se obtuvo un alfa de Cronbach, con un valor numérico $\alpha=.843$, así como la prueba de las dos mitades de Guttman, cuyo resultado ha sido de .792 . Ambos valores, se consideran óptimos, e indican una fiabilidad alta del cuestionario construido.

\section{Análisis de datos}

Para llevar a cabo el análisis de datos, se utilizó el paquete estadístico SPSS v25, así como el programa Rstudio en su versión 1.2.5. En primer lugar, se procedió a llevar a cabo el análisis descriptivo, con la finalidad de conocer las percepciones de los estudiantes de las competencias profesionales que debe reunir un docente. Seguidamente, previa aplicación del test de Kolmogórov-Smirnov con la corrección de Lilliefords sobre normalidad y la prueba de Levene de homogeneidad de varianzas, se aplicó los estadísticos $U$ de MannWhitney y $W$ de Wilcoxon para conocer si existían diferencias significativas en las respuestas de los sujetos en torno a la variable independiente género. Por último, se aplicó el test de Pearson sobre correlación de variables, con el objeto de conocer si existen relaciones proporcionales entre las variables que componen el estudio.

\section{Resultados}

En primera instancia, el análisis de los estadísticos descriptivos permitió reflejar que el alumnado le otorga gran importancia a las distintas competencias profesionales que debe tener un profesor a la hora de ejercer su labor didáctico-pedagógica (tabla 2). Esta afirmación queda refleja, no solamente en las medias aritméticas obtenidas, que reflejan que la importancia que le dan los estudiantes a las competencias profesionales del docente oscila entre "Mucho" y "Bastante".

Destacan especialmente el ítem 6 seguidos del ítem 7 y el ítem 12, que presenta los valores de media aritmética más próximos a 1 , y que, por tanto, indican un mayor grado de importancia hacia la afirmación descrita en la variable. El nivel de dispersión de las respuestas no es excesivo, lo cual permite inducir la idea acerca de la no existencia de outliers en la distribución de datos.

Respecto a los estadísticos de forma (asimetría y curtosis), destacan la asimetría presentada en los ítems 6,7 y 13 , que se encuentran sus valores respectivos por encima de 1, presentando por tanto una distribución en la que los resultados obtenidos se encuentran por encima de la media aritmética obtenida, y la distribución quedará sesgada en la parte derecha. En el caso de la curtosis, tal y como se puede observar en la tabla 2, gran parte de los ítems analizados (2, 7, 11, 12, 13, y en especial, el 6$)$, presentan un coeficiente de curtosis positivo, tratándose por tanto de curvas leptocúrticas, en la que los datos se encuentran muy concentrados en torno a la media. En el caso los ítems 1,3,4,5,8,9 y 10, presenta un 
coeficiente de curtosis negativo, tratándose por tanto de curvas platicúrticas, y así mostrando una concentración de datos más escasa en torno a la media.

Tabla 1.

Estadísticos descriptivos variables dependientes del estudio

\begin{tabular}{ccccc}
\hline Ítem & Media & Desv.Típ & Asimetría & Curtosis \\
\hline 1 & 1,78 & .670 & .431 & -.168 \\
2 & 1.54 & .633 & .978 & .972 \\
3 & 1.66 & .681 & .638 & -.306 \\
4 & 1.87 & .715 & .239 & -.884 \\
5 & 1.56 & .654 & .792 & -.205 \\
6 & 1.43 & .897 & 6.316 & 62.132 \\
7 & 1.50 & .653 & 1.076 & .467 \\
8 & 1.74 & .692 & .522 & -.299 \\
9 & 2.12 & .731 & .042 & -.630 \\
10 & 2.45 & .827 & .005 & -.542 \\
11 & 1.77 & .746 & .693 & .021 \\
12 & 1.50 & .679 & 1.193 & .893 \\
13 & 1.51 & .668 & 1.152 & .918 \\
\hline
\end{tabular}

A continuación, para la realización de la comparación entre medias, resultó preciso el cálculo de manera previa de test para comprobar el carácter paramétrico o no de la distribución de datos. Para ello, en primer lugar, se calcula el índice de normalidad a través de la prueba de Kolmogórov-Smirnov con la corrección de Lilliefords en la que obtenemos un valor significativo $(p<0.01)$, lo cual denota que la distribución no sigue una tendencia normal o de campana de Gauss. A su vez, se remite a la comprobación del grado de homocedasticidad u homogeneidad de varianzas, a través de la prueba de Levene en al que se obtuvieron que la totalidad de los ítems no presentaron significatividad, y por lo tanto confirman que un carácter no paramétrico en la distribución.

En base a esto, se procedió a la aplicación de la prueba $U$ de Mann-Whitney y $W$ de Wilcoxon en torno a la variable independiente género. Los resultados presentaron que no existen grandes diferencias significativas entre los géneros en las variables estudiadas. Sin embargo, en el caso de tres variables (ítem 3, 5 y 12) la significación presentó resultados óptimos, demostrando que existen diferencias en las percepciones del estudiantado en función del género.

Tabla 2.

Estadísticos U-Mann-Whitney y W de Wilconxon

\begin{tabular}{|c|c|c|c|c|c|c|c|}
\hline \multicolumn{2}{|c|}{ Ítem-Sexo } & Rango & Suma de & U Mann- & W. & $\bar{Z}$ & $P$ \\
\hline \multirow[t]{2}{*}{ I.1 } & Hom & 209.67 & 30192 & 18551 & 54062 & -.583 & .560 \\
\hline & Muj & 203.24 & 54062 & & & & \\
\hline \multirow[t]{2}{*}{1.2} & Hom & 204.74 & 29483 & 19043 & 29483 & -.108 & .914 \\
\hline & Muj & 205.91 & 54772 & & & & \\
\hline \multirow[t]{2}{*}{1.3} & Hom & 215.65 & 31053 & 17690 & 53201 & -1.407 & .023 \\
\hline & Muj & 200.01 & 53201 & & & & \\
\hline \multirow[t]{2}{*}{1.4} & Hom & 202.55 & 29166 & 18726 & 29166 & -.404 & .687 \\
\hline & Muj & 207.10 & 55088 & & & & \\
\hline \multirow[t]{2}{*}{1.5} & Hom & 220.51 & 31753 & 16991 & 52502 & -2.115 & .034 \\
\hline & Muj & 197.38 & 52502 & & & & \\
\hline
\end{tabular}




\begin{tabular}{|c|c|c|c|c|c|c|c|}
\hline \multirow[t]{2}{*}{1.6} & Hom & 209.05 & 30103 & 18641 & 54152 & -.539 & .590 \\
\hline & Muj & 203.58 & 54152 & & & & \\
\hline \multirow[t]{2}{*}{1.7} & Hom & 207.25 & 29843 & 18900 & 54411 & -.252 & .801 \\
\hline & Muj & 204.55 & 54411 & & & & \\
\hline \multirow[t]{2}{*}{1.8} & Hom & 205.06 & 29528 & 19088 & 29528 & -.061 & .951 \\
\hline & Muj & 205.74 & 54727 & & & & \\
\hline \multirow[t]{2}{*}{1.9} & Hom & 206.44 & 28991 & 19016 & 54527 & -.129 & .897 \\
\hline & Muj & 204.99 & 55263 & & & & \\
\hline \multirow[t]{2}{*}{ I.10 } & Hom & 201.33 & 30778 & 18551 & 28991 & -.560 & .576 \\
\hline & Muj & 207.76 & 53476 & & & & \\
\hline \multirow[t]{2}{*}{ I.11 } & Hom & 213.74 & 30778 & 17965 & 53476 & -1.129 & .259 \\
\hline & Muj & 201.04 & 53476 & & & & \\
\hline \multirow[t]{2}{*}{ I.12 } & Hom & 214.68 & 30914 & 17830 & 53341 & -1.326 & .031 \\
\hline & Muj & 200.53 & 53341 & & & & \\
\hline \multirow[t]{2}{*}{$\mathrm{I} .13$} & Hom & 213.36 & 30724 & 18019 & 53530 & -1.130 & .185 \\
\hline & Muj & 201.24 & 53530 & & & & \\
\hline
\end{tabular}

Nota: Z= Estadístico de Wilconxon; $\mathrm{P}: \mathrm{p}$ valor

Finalmente, la aplicación del test de Pearson, permitió conocer diferentes relaciones de correlación entre las variables del estudio. En primer lugar, se observa que, a excepción de la relación entre 1.6 e i.10, se dilucidan relaciones correlativas positivas entre todo el conjunto de variables. En suma, todas las variables presentaron niveles de correlación leve, a excepción del I.10 con el I.6. En concreto, se observan relaciones más severas entre I.5 e I.13, I.7 e I.8 o I.12 e I.13. Todas ellas presentaron un nivel de significación óptimo ( $p<.05)$ 


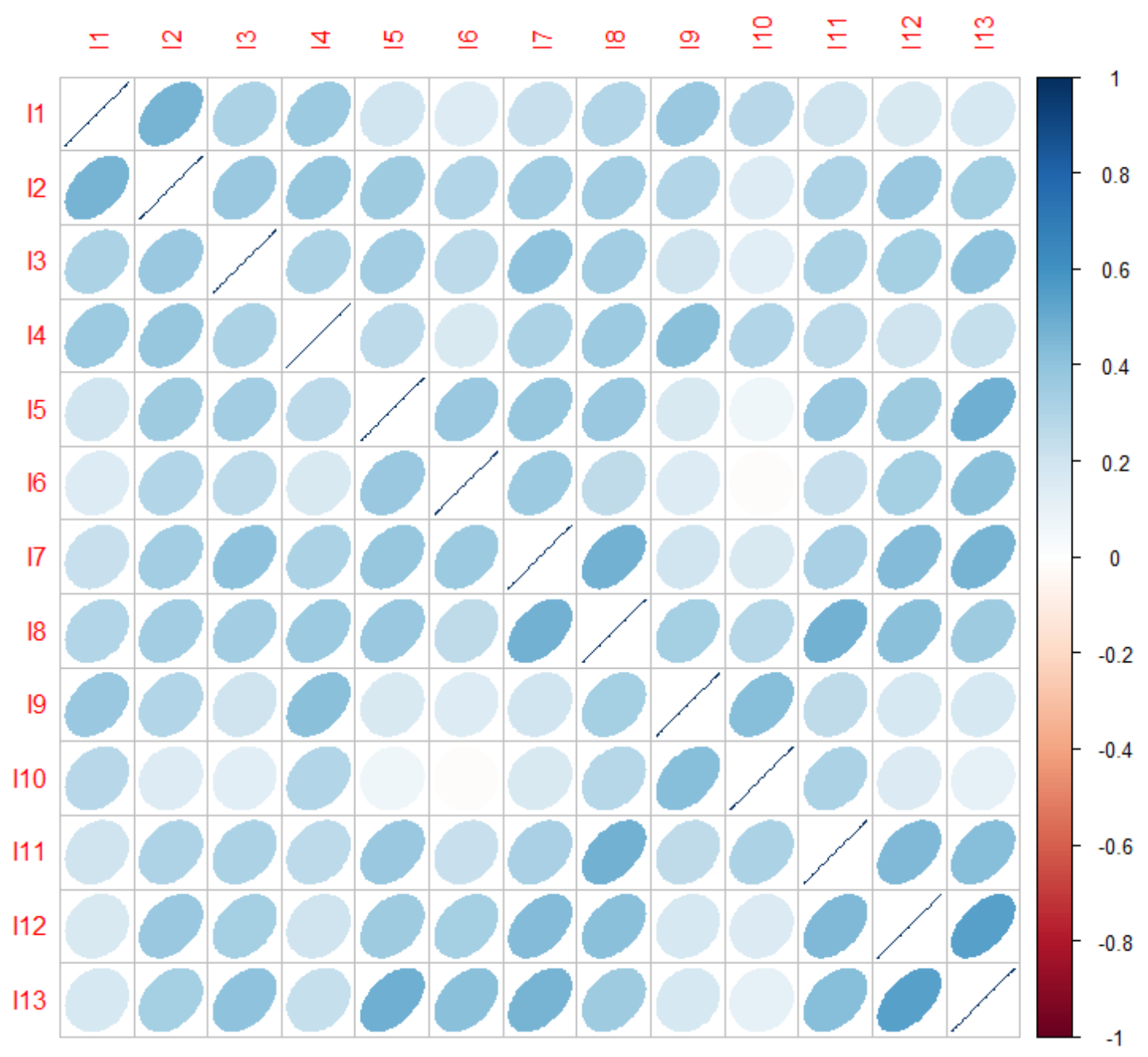

Figura 1. Correlación entre variable.

\section{Discusión}

Con el presente estudio se planteó como objetivo conocer las percepciones que los estudiantes acerca diferentes competencias profesionales del docente, previo comienzo del curso del Máster oficial de Profesorado de Educación Secundaria Obligatoria y Bachillerato, Formación profesional y Enseñanza de Idiomas de la Universidad de Granada. Asimismo, se planteó conocer si existían diferencias significativas respecto al género en las respuestas obtenidas, y si existían relaciones correlaciónales entre las variables.

Los resultados obtenidos revelaron la importancia que el estudiantado otorga a las competencias profesionales del docente, al igual que en el estudio de Terigi (2009). En esta misma línea, se observó que los estudiantes otorgan un mayor grado de importancia a que el docente adquiera estrategias que permitan estimular al estudiante de cara al proceso de aprendizaje, el fomento de habilidades críticas, así como también aquellas competencias asociadas a la creación de un clima de confianza en el aula, de interacción y comunicación en el aula. Estos resultados muestran una clara tendencia de que los futuros profesores abogan por una necesidad de que la figura del docente que conozca nuevas medidas para motivar a sus estudiantes, apostando por una enseñanza emprendedora e innovadora, olvidando concepciones de enseñanza tradicionales (Muñoz, 2003). 
En este sentido, los resultados obtenidos confirman que el alumnado del Máster de Secundaria valora positivamente las competencias que hacen referencia a la necesidad de saber planificar, desarrollar y evaluar el proceso de enseñanza y aprendizaje potenciando procesos educativos que faciliten la adquisición de las competencias propias de las respectivas enseñanzas, atendiendo al nivel y formación previa de los estudiante; diseñar y desarrollar espacios de aprendizaje con especial atención a la equidad, la educación emocional y en valores, la igualdad de derechos y oportunidades; fomentar y garantizar el respeto a los derechos humanos y a los principios de accesibilidad universal, igualdad, no discriminación y los valores democráticos y de la cultura de la paz, coincidiendo con Fernández y Bueno (2016). Así pues, se trata de concepciones que abarca no solamente una formación curricular estricta, sino que se trata de una educación integral, especialmente dirigida a formar personas.

En consonancia a esta idea, el estudiantado valoró vagamente el conocimiento de los contenidos curriculares de las materias relativas a la especialización docente correspondiente, así como el cuerpo de conocimientos didácticos en torno a los procesos de enseñanza y aprendizaje respectivos; la búsqueda, obtención, proceso y comunicación de la información transformándola en conocimiento y aplicándola en los procesos; concreción del currículo que se vaya a implantar en un centro docente participando en la planificación colectiva del mismo; desarrollo de las funciones de tutoría y de orientación de los estudiantes de manera colaborativa y coordinada, participando en la evaluación, investigación e innovación de los procesos de enseñanza y aprendizaje; conocimiento de la normativa y organización institucional del sistema educativo y modelos de mejora de la calidad con aplicación a los centros de enseñanza; conocimiento y análisis de las características históricas de la profesión docente, su situación actual, perspectivas e interrelación con la realidad social de cada época (Martín, Pastor y Oliva, 2019; Lauermann y Konig).

Asimismo, el análisis de las pruebas de comparación de medias permitió verificar que no existían diferencias significativas en las respuestas obtenidas en función del género del estudiante. Finalmente, el establecimiento de relaciones correlaciónales permitió localizar algunas relaciones fuertes entre las variables, especialmente entre aquellas variables dedicadas a la puesta en valor de la educación emocional, la enseñanza humanitaria y en diversidad; la trascendencia de la orientación educativa y la acción tutorial en el aula o; la importancia de una enseñanza que promueva el desarrollo de habilidades de pensamiento crítico con la importancia de tornar el proceso de enseñanza hacia la enseñanza de destrezas emprendedoras.

\section{Conclusiones}

La figura del docente continúa siendo un campo de conocimiento que resulta de interés para la comunidad investigadora. Con el paso de los años, se percibe una evolución en torno las competencias profesionales que el docente debe poseer, que a pesar de seguir teniendo en cuenta el marco curricular y didáctico, torna hacia una enseñanza que prioriza lo emocional, la tutoría y la orientación educativa como base de la acción docente.

A través de estudio, se ha pretendido establecer un acercamiento acerca de las percepciones que presentan los estudiantes del Máster de Secundaria sobre aquellas competencias que debe presentar un docente. Resulta fundamental que los futuros profesores conozcan cuales son las competencias que debe de disponer un profesor para confrontar con eficacia las futuras demandas que le requerirá el contexto laboral.

Referente a las limitaciones del estudio, se encuentra en la conformación del instrumento, que podría haber contado con un mayor número de variables que profundizaran en más 
competencias profesionales que abarca el docente. Por otro lado, como futuras líneas de investigación, se propone a la comunidad investigadora a seguir contribuyendo al ámbito de la formación del profesorado a través de la elaboración de experiencias con futuros profesores y el conocimiento de las competencias profesionales, a través de estudios experienciales con futuros profesores en los que se midan sus actitudes y percepciones acerca de aquellas competencias que debe disponer un docente a la hora de enfrentarse a un aula.

En conclusión, la formación del profesorado continúa siendo un pilar fundamental en el panorama educativo actual. Resulta prioritario que la formación desde las etapas educativas superiores, se fomente una praxis adecuada que dote al estudiantado de nociones y competencias, de tal manera que puedan responder con eficacia a las futuras demandas que le exigirá su profesión.

\section{Referencias}

Acebedo-Afanador, M. J., Aznar-Díaz, I. e Hinojo-Lucena, F. J. (2017). Instrumentos para la Evaluación del Aprendizaje Basado en Competencias: Estudio de caso. Información tecnológica, 28(3), 107-118. https://doi.org/10.4067/s0718-07642017000300012

Fernández, J. T. y Bueno, C. R. (2016). Evaluation of professional competences in higher education: challenges and implications. Educación XXI, 19(1), 17-38. doi:10.5944/educXX1.12175

Friedman, I. A. (2016). Being a teacher: altruistic and narcissistic expectations of pre-service teachers. Teachers and Teaching, 22(5), 625-648. doi:10.1080/13540602.2016.1158469

Hernández, R., Fernández, C. y Baptista, P. (2016). Research methodology (6th edition). Mexico: McGraw-Hill.

Hill, F. M. (1995). Managing service quality in higher education: The role of the student as primary consumer. Quality Assurance in Education, 3(3), 10-21. doi:10.1108/09684889510093497

Kass, E., \& Miller, E. C. (2018). Career choice among academically excellent students: Choosing teaching career as a corrective experience. Teaching and Teacher Education, 73, 90-98. doi:10.1016/j.tate.2018.03.015

Khattab, N. (2015). Students' aspirations, expectations and school achievement: what really matters? British Educational Research Journal, 41(5), 731-748. doi:10.1002/berj.3171

Konig, J. (2013). First comes the theory, then the practice? On the acquisition of general pedagogical knowledge during initial teacher education. International Journal of Science and Mathematics Education, 11(4), 999-1028. doi:10.1007/s10763-013-9420-1

Lauermann, F., \& Konig, J. (2016). Teachers' professional competence and wellbeing: Understanding the links between general pedagogical knowledge, self-efficacy and burnout. Learning and Instruction, 45, 9-19. doi:10.1016/j.learninstruc.2016.06.006

López-Roldán, P., \& Fachelli, S. (2015). Metodología de la investigación social cuantitativa. Bellaterra (Cerdanyola del Vallès): Dipòsit Digital de Documents, Universitat Autònoma de Barcelona.

Martín, L. C., Pastor, M. S., \& Oliva, F. J. C. (2019). Competencias docentes en la formación inicial del profesorado de educación física. Retos: nuevas tendencias en educación física, deporte y recreación, (35), 284-288. https://doi.org/10.35376/10324/39394 
Moreno Iglesias, M., Casanova Moreno, M. D. L. C., Socarrás, M. M., Álvarez García, B. I., \& Tabares Arévalo, R. (2019). El currículo, las competencias profesionales del docente: un reto de las carreras pedagógicas y médicas. Revista de Ciencias Médicas de Pinar del Río, 23(1), 108-118. https://doi.org/10.11565/arsmed.v20i1.601

Muñoz, R. (2003). Competencias profesionales del docente en la sociedad del siglo XXI. Organización y Gestión Educativa: Revista Del Fórum Europeo De Administradores De La Educación. 11(1) 4-7. https://doi.org/10.4272/84-9745-093-0.ch18

Ortiz. (2002). Las competencias profesionales del ingeniero mecánico. Una alternativa de diseño curricular. Universidad de Puerto Rico.

Otzen, T., \& Manterola, C. (2017). Técnicas de Muestreo sobre una Población a Estudio. International Journal of Morphology, 35(1), 227-232. https://doi.org/10.4067/s071795022017000100037

Paiva, A. D. F., Romero, J. M., \& Gómez, G. (2018). Desarrollo profesional y formación continua de profesores: un análisis del contexto de la educación en Angola. Innoeduca: international journal of technology and educational innovation, 4(1), 71-78.

Pesquero, E., Martín, M. E. S., Ballesteros, M. G., del Pozo, R. M., González, S. G., Collazos, J. C., y Nieto, P. V. (2008). Las competencias profesionales de los maestros de Primaria. Revista española de pedagogía, 447-466.

Ramírez, E. S., de Pozo, R. M., Rivero, A., \& Porlán, R. (2013). Expectativas y concepciones de los estudiantes del MAES en la especialidad de Ciencias. Revista Eureka sobre enseñanza y divulgación de las ciencias, 10,496-513. https://doi.org/10.25267/rev_eureka_ensen_divulg_cienc.2013.v10.iextra.02

Rego, M. A. S., \& Moledo, M. L. (2015). Training of Secondary Education Teachers: thinking about the reconstruction of the university project. Revista Española De Pedagogía, 73(261), 263-281.

Sander Paul, Stevenson Keith, King Malcolm, Coates David. University students' expectations of teaching. Studies in Higher Educcation, 25, 2000(3), 309-23. https://doi.org/10.1080/03075070050193433

Serrano, R., \& Pontes, A. (2015). Expectativas ante la formación inicial entre el alumnado del Máster de Profesorado en Enseñanza Secundaria. Revista de Investigación Educativa, 33(2), 489-505. https://doi.org/10.6018/rie.33.2.203471

Telford, R., \& Masson, R. (2005). The congruence of quality values in higher education. Quality Assurance in Education, 13(2), 107-119. doi:10.1108/09684880510594364

Terigi, F. (2009). Initial teacher training in Secondary Education: needs to improve, acknowledgement of its limits. Revista De Educación (350), 123-144.

Voss, R., Gruber, T., \& Szmigin, I. (2007). Service quality in higher education: The role of student expectations. Journal of Business Research, 60(9), 949-959. doi:10.1016/j.jbusr.2007.01.020 